\title{
Biomembrane mimics and their roles in anti-bacterial drug discovery
}

\author{
Xiaohui Huand Kin Tam* \\ Drug Development Core, Faculty of Health Sciences, University of Macau, Macau, China \\ *Corresponding Author: E-mail: kintam@umac.mo; Tel.: +853-8822-4988; Fax: +853-8822-2314
}

Received: February 17, 2017; Revised: February 19, 2017; Published: March 25, 2017

\begin{abstract}
Cell membrane is at the frontline of the battle between pathogenic microbe and host. A thorough understanding of bacterial membrane is fundamental to tackle infection disease. Membrane mimetic provides a powerful tool for mechanistic investigation of drug-membrane interaction. Herein, we summarized major features of bacterial and mammalian cell in context of antibacterial therapy. Many details were given to model membranes and their application in mechanistic studies. Current challenge in antibacterial therapy and perspective of membrane mimics in antibacterial drug discovery were also provided.
\end{abstract}

\section{Keywords}

Drug-membrane interaction; mode of action; liposome; supported lipid bilayer; resistance.

\section{Introduction}

Cell membrane defines the physical boundary of a cell from outside environment. It contains lipids, proteins and carbohydrates, which usually arrange into structure unique to specific cell type and are critical to corresponding function. Of all kinds of membrane components, lipids are relatively homogenous and better studied. Lipids are amphipathic molecules with a polar or charged head group and a hydrophobic acyl chain tail. They tend to form head-by-head, tail-to-tail bilayer structure in cell membrane to extrude water, salt and other hydrophilic molecules and maintain low entropy state. This ordered bilayer structure serves as the membrane framework, on which proteins and carbohydrates carry out their designated biological functions. As the target of more than half of all modern drugs [1], membrane proteins interact with surrounding lipid through their transmembrane domain and are vital to variety of cell function, including signal transduction, transporting, enzymatic catalysis and cell adhesion [2]. Carbohydrates in plasma membrane, predominantly glycoproteins but also with some glycolipids, are generally found on the extracellular side and play significant roles in many biological events such as cell adhesion and immunological response. As diverse as the membrane composition is, these components are not randomly or evenly distributed around the cell sphere. They tend to form distinct regions or microdomains, which contain specific type of lipids, glycolipids and proteins within fluid bilayer and perform unique biological function such as signal transduction $[3,4]$. 
Studying biological membrane is always a challenging task because of its complicated composition and fluidic structure. These difficulties could be partially circumvented by using simplified lipids-based model systems, such as vesicles, micelles, bicelles, nanodiscs and bilayers. Through control of the lipid content and buffer conditions (salt concentration, $\mathrm{pH}$ etc.), model systems can be utilized to explore interaction between membranes and exogenous objects (such as drugs) quantitatively.

In this article, we will touch specifics about bacterial and mammalian cell membrane in context of antibacterial therapy. We will then discuss the development of membrane models and their application in studies pertaining to biological function of the membrane. We will close the review with current challenge in antibacterial therapy and perspective of membrane mimics.

\section{Bacterial and mammalian membrane}

Although lipids in plasma membrane can be schematized as bilayer for simplicity, its composition and structure could differ greatly among species, cells from different organisms and tissues. In general, grampositive bacteria contain a single cell membrane surrounded by a thick layer of peptidoglycan (cell wall), while gram-negative bacteria plasma membrane is made up of two layer membrane (inner- and outer) encompassed by a thin layer of peptidoglycan. Membrane of the model bacterium Escherichia coli is made up of three major phospholipids: $75 \%$ phosphatidylethanolamine (PE), $20 \%$ phosphatidylglycerol (PG) and $\sim 5 \%$ cardiolipin (CL) [5]. But this cannot be generalized to all bacteria, as diversified lipids can be found in different microorganisms, or even in the same species in different environment [6].

In contrast to bacterial membrane, mammalian membrane is void of cell wall and exposed directly to surrounding environment, which thus demands more stringent regulation. Mammalian membranes of different cell types or from distinct tissues could vary significantly in its fluidity, which is believed to be modulated at least partially by the content of cholesterol [4], an integral lipid unique to animal cell membrane. Asymmetric distribution of lipid components on different sides of individual cell is another remarkable feature of multicellular organism cell membrane. Structural and compositional difference between bacteria and mammalian cell membrane represents also a big opportunity in antibacterial therapy for patient.

\section{Antimicrobial actions that are related to bacterial membranes}

Pathogenic bacteria pose a major threaten to public health, especially in under-developed regions of the world. Large scale production and use of antibiotic penicillin during the World War II is a hallmark of modern medicine. Since then, a plethora of antimicrobial agents have been developed and commercialized, playing significant roles in improvement of human health, highlighted by gradual increasing of average life span worldwide.

Depending on their structure and target sites within microorganism, different antibacterial drugs have distinct modes of action. Modes of action related to bacterial membrane are inhibition or block of cell wall synthesis, inhibition of membrane protein or lipid synthesis and interference of cell membrane function. As aforementioned, bacterial membrane is surrounded by a layer of peptidoglycan cell wall. This unique feature rendered a great interest in searching for glycopeptide and lipoglycopeptide, which mimic bacterial cell wall synthesis substrates or bind to synthesis intermediates, inhibiting cell wall synthesis [7], while having little effect on host cells. Some antibacterial peptides [8,9], peptidomimics [10] and small proteins [11] could also interact with or insert into cell membrane, where they interfere with membrane formation or alter local membrane structure, thus reducing bacteria growth or reproduction. Interestingly, some 
pathological microorganisms could also as a countermeasure secrete protein toxins which could attack host cells by forming pores on their membranes $[12,13]$. Membrane is the combating field of the war between pathogens and their hosts, which also make mechanistic study on biological membrane fundamental to antibacterial therapy development.

\section{Current biomimetic membrane models and applications}

The complexity of biological membrane makes it always difficult or impractical in a lot of case to study the membrane intact. A variety of membrane models has been developed over decades to overcome the obstacle.

Vesicles (liposome) are the most commonly used biological mimics as they are relatively easy to prepare and handle. Vesicle sphere consists of self-assembled round lipid-bilayer(s) which mimic plasma membrane. Enclosed aqueous compartment resembles cytoplasma can be used to incorporate diverse materials of interest (including drugs). Depending on its size and structure, vesicle can be either smallunilamellar (SUV), large-unilamellar (LUV) or multilamellar (MLV). A variety of biophysical methods have been employed to study the interaction between drug and membrane mimicked by lipid vesicle. Simple mathematic model of drug diffusion into or release from cell could be derived from those studies. In addition to its application in the mechanistic study of membrane, controlled inner environment of liposomes have been used to deliver charged drugs [14,15] through membrane [16].

Micelles are aggregates of surfactant molecules dispersed in a liquid. The stability of colloid solution was established by the repulsion forces between hydrophobic surfactant tails and hydrophilic solvent molecules. Lipid molecules can be used as surfactant to form micelle and to dissolve membrane protein in the structural studies [17-19]. However, the application of micelles as membrane mimics is limited by its small size and a single layer structure. In disc-shaped bicelles, lipid hydrophobic tails pack against each other as bilayer in the center and form an amphipathic, micelle-like assembly at the edge. Bicelles are much smaller than vesicles, and are ideal in the experiments where the larger liposomes are not an option [20-22]. Nanodiscs are patch of lipid bilayer whose edge is wrapped with an amphipathic coating protein [23]. Compared to bicelles and micelles, nanodiscs are more stable and uniform in size (depending on the length of coating protein). Membrane protein of interest (such as drug target) can be inserted into nanodiscs and examined both biophysically and functionally [24].

Other than being freely mobile in aqueous solution, lipid-based membranes can also be studied while attached to device or to supporting materials. The first model developed of this kind is a black lipid membrane (BLM) $[25,26]$, where lipid bilayer is formed within an aperture on a thin layer of hydrophobic material such as Teflon. Black lipid membrane has been employed to study membrane properties (electrical potential [27], structure [28] and function [29]), lipid-protein interaction [30], peptides or protein diffusion across membrane [31]. Application of micro-black lipid membrane technology had led to development of high throughput drug permeability assay system PAMPA (Parallel Artificial Membrane Permeation Assay) [32]. Lipid bilayer can also be attached to the surface of solid material to form a stable membrane mimic called supported lipid bilayer (SLB). Great stability and surface planarity of supported lipid bilayer make it especially useful for techniques requiring direct physical contact (e.g. atomic force microscopy (AFM) [33] and electrode-supported lipid nanoassemblies [34]) or rigidly-supported planar surface (e.g. total internal reflection fluorescence microscopy (TIRF) [35] and surface plasmon resonance (SPR) [36]). Supported lipid bilayer has another advantage in that, in light of the lipid rafts concept, lipids can be patterned into isolated regions through design of the surface [37]. 
Beside lipids, biological membranes also contain variety of protein and carbohydrates. Thus, extra care must be taken when interpreting the studies obtained from lipid-based membrane mimics. In more advanced model systems, membrane proteins can also be reconstituted into artificial lipid bilayer to simulate protein function in lipid environment [34].

\section{Challenge and perspective}

One of the biggest problems in the antibacterial chemotherapy field is development of pathogen resistance to antibiotics [38]. Infectious pathogen could quickly develop resistance to drugs applied on them by either mutating into new strains to get around the drug blocking pathway or inducing counting mechanism to remove the drug [39]. On the other hand, development of novel antibiotics has been stagnant since a half century ago [40]. A thorough understanding of the mechanism of drug action, metabolism and transportation is critical to develop new drugs to cope with antibiotics resistance. Model membrane study is fundamental to elucidating these mechanisms, upon which development of agents with novel [41-43] and/or multiple $[39,44,45]$ mode(s) of action poses some most promising strategies to bring us new tools to combat microbial infection diseases.

\section{Conclusions}

Cell membranes are at the frontlines of the battle field between pathogenic microbe and host. Mechanistic study of the membrane is crucial for a thorough understanding of host-pathogen interaction, which could lead to discovery of new drug for efficient infection control. Biomembrane mimics are powerful tools to gain mechanistic insight of drug-membrane interaction by simplifying otherwise complicated membrane structure with controlled components. Multiple type of biomimetic membrane have been developed to meet variety of needs in mechanistic studies.

Acknowledgements: We thank the financial support from the University of Macau (grant no.: SRG201300055-FHS).

\section{References}

[1] J.P. Overington, B. Al-Lazikani, A.L. Hopkins, Nat. Rev. Drug Discov. 5 (2006) 993-996.

[2] M.S. Almén, K.J.V. Nordström, R. Fredriksson, H.B. Schiöth, BMC Biol. 7 (2009) 50.

[3] D. Lingwood, K. Simons, Science 327 (2010) 46-50.

[4] K. Simons, D. Toomre, Nat. Rev. Mol. Cell Biol. 1 (2000) 31-39.

[5] C. Sohlenkamp, O. Geiger, FEMS Microbiol. Rev. (F. Narberhaus, Ed.) 40 (2016) 133-159.

[6] I.M. López-Lara, O. Geiger, Biochim. Biophys. Acta - Mol. Cell Biol. Lipids. (2016) doi: 10.1016/j.bbalip.2016.10.007.

[7] D. Kahne, C. Leimkuhler, W. Lu, C. Walsh, Chem. Rev. 105 (2005) 425-448.

[8] M. Zasloff, Nature 415 (2002) 389-395.

[9] K.A. Brogden, Nat. Rev. Microbiol. 3 (2005) 238-250.

[10] T. Velkov, P.E. Thompson, R.L. Nation, J. Li, J. Med. Chem. 53 (2010) 1898-1916.

[11] S. Mukherjee, H. Zheng, M.G. Derebe, K.M. Callenberg, C.L. Partch, D. Rollins, D.C. Propheter, J. Rizo, M. Grabe, Q.-X. Jiang, L.V. Hooper, Nature 505 (2014) 103-107.

[12] C.J. Rosado, S. Kondos, T.E. Bull, M.J. Kuiper, R.H.P. Law, A.M. Buckle, I. Voskoboinik, P.I. Bird, J.A. Trapani, J.C. Whisstock, M.A. Dunstone, Cell. Microbiol. 10 (2008) 1765-1774. 
[13] R.H.P. Law, N. Lukoyanova, I. Voskoboinik, T.T. Caradoc-Davies, K. Baran, M.A. Dunstone, M.E. D’Angelo, E.V. Orlova, F. Coulibaly, S. Verschoor, K.A. Browne, A. Ciccone, M.J. Kuiper, P.I. Bird, J.A. Trapani, H.R. Saibil, J.C. Whisstock, Nature 468 (2010) 447-451.

[14] T.M. Allen, P.R. Cullis, Adv. Drug Deliv. Rev. 65 (2013) 36-48.

[15] V.P. Torchilin, Nat. Rev. Drug Discov. 4 (2005) 145-160.

[16] A.S. Ulrich, Biosci. Rep. 22 (2002) 129-150.

[17] N. Sanghera, T.J. Pinheiro, Protein Sci. 9 (2000) 1194-1202.

[18] C.M. Franzin, P. Teriete, F.M. Marassi, J. Am. Chem. Soc. 129 (2007) 8078-8079.

[19] N.P. Barrera, N. Di Bartolo, P.J. Booth, C.V. Robinson, Science 321 (2008) 243-246.

[20] S. Faham, J.U. Bowie, J. Mol. Biol. 316 (2002) 1-6.

[21] S. Faham, G.L. Boulting, E.A. Massey, S. Yohannan, D. Yang, J.U. Bowie, Protein Sci. 14 (2005) 836840.

[22] R. Ujwal, J. Abramson, J. Vis. Exp. 59 (2012) e3383.

[23] A. Nath, W.M. Atkins, S.G. Sligar, Biochemistry 46 (2007) 2059-2069.

[24] T.H. Bayburt, S.G. Sligar, FEBS Lett. 584 (2010) 1721-1727.

[25] P. Mueller, D.O. Rudin, H.T. Tien, W.C. Wescott, Nature 194 (1962) 979-980.

[26] H.T. Tien, S. Carbone, E.A. Dawidowicz, Nature 212 (1966) 718-719.

[27] S. Ozeki, H. Kurashima, M. Miyanaga, C. Nozawa, Langmuir 16 (2000) 1478-1480.

[28] R.S. Ries, H. Choi, R. Blunck, F. Bezanilla, J.R. Heath, J. Phys. Chem. B 108 (2004) 16040-16049.

[29] P. van Gelder, F. Dumas, M. Winterhalter, Biophys. Chem. 85 (2000) 153-167.

[30] R. Smekal, H.P. Ting, L.G. Augenstein, H.T. Tien, Science 168 (1970) 1108-1109.

[31] K. Wei, A. Neef, Q. Van, S. Kramer, I. Gregor, J. Enderlein, Biophys. J. 105 (2013) 455-462.

[32] M. Kansy, F. Senner, K. Gubernator, J. Med. Chem. 41 (1998) 1007-1010.

[33] O. Domenech, Y.F. Dufrêne, F. van Bambeke, P.M. Tukens, M.-P. Mingeot-Leclercq, Biochim. Biophys. Acta 1798 (2010) 1876-1885.

[34] M. Wang, W. Zhan, Acc. Chem. Res. 49 (2016) 2551-2559.

[35] J.M. Moran-Mirabal, J.B. Edel, G.D. Meyer, D. Throckmorton, A.K. Singh, H.G. Craighead, Biophys. J. 89 (2005) 296-305.

[36] M.A. Cooper, D.H. Williams, Chem. Biol. 6 (1999) 891-899.

[37] J.T. Groves, N. Ulman, S.G. Boxer, Science 275 (1997) 651-653.

[38] L.L. Silver, Clin. Microbiol. Rev. 24 (2011) 71-109.

[39] F. Guilhelmelli, N. Vilela, P. Albuquerque, L.S. Derengowski, I. Silva-Pereira, C.M. Kyaw, Front. Microbiol. 4 (2013) 353.

[40] M.A. Fischbach, C.T. Walsh, Science 325 (2009) 1089-1093.

[41] S.C.P. Williams, Proc. Natl. Acad. Sci. U. S. A. 111 (2014) 11227-11229.

[42] D.G. Brown, T. Lister, T.L. May-Dracka, Bioorg. Med. Chem. Lett. 24 (2014) 413-418.

[43] L.L. Ling, T. Schneider, A.J. Peoples, A.L. Spoering, I. Engels, B.P. Conlon, A. Mueller, T.F. Schäberle, D.E. Hughes, S. Epstein, M. Jones, L. Lazarides, V.A. Steadman, D.R. Cohen, C.R. Felix, K.A. Fetterman, W.P. Millett, A.G. Nitti, A.M. Zullo, C. Chen, K. Lewis, Nature 517 (2015) 455-459.

[44] L. Otvos, C. Snyder, B. Condie, P. Bulet, J.D. Wade, Int. J. Pept. Res. Ther. 11 (2005) 29-42.

[45] V. Yarlagadda, S. Samaddar, K. Paramanandham, B.R. Shome, J. Haldar, Angew. Chem. Int. Ed. Engl. 54 (2015) 13644-13649. 